\title{
Engagement e Inteligencia emocional en estudiantes de una universidad privada en la ciudad de Arequipa
}

\section{Engagement and Emotional Intelligence in Students of a Private University in the City of Arequipa}

\author{
Dennis Arias-Chávez* \\ Universidad Continental, Arequipa, Perú \\ ORCID: https://orcid.org/0000-0003-1500-8366 \\ Mervi P. Vera-Buitrón ${ }^{D}$ \\ Universidad Privada del Norte, Lima, Perú \\ ORCID: https://orcid.org/0000-0001-5387-1983 \\ Teresa Ramos-Quispe \\ Universidad Nacional de San Agustín de Arequipa, Arequipa, Perú \\ ORCID: https://orcid.org/0000-0003-4607-4745 \\ Segundo Pérez-Saavedra \\ Pontificia Universidad Católica del Perú, Lima, Perú \\ ORCID: https://orcid.org/0000-0002-2366-6724
}

Recibido 25-07-19 Revisado 13-08-19 Aprobado 07-12-19 En línea 10-12-19

*Correspondencia

Email: darias@continental.edu.pe
Citar como:

Arias-Chávez, D., Vera-Buitrón, M., Ramos-Quispe, T., \& Pérez-Saavedra (2020). Engagement e Inteligencia emocional en estudiantes de una universidad privada en la ciudad de Arequipa. Propósitos y Representaciones, 8(1), e423. doi: http://dx.doi.org/10.20511/pyr2020.v8n1.423 


\section{Resumen}

El objetivo del presente estudio fue determinar la relación entre el engagement y la inteligencia emocional en estudiantes hombres y estudiantes mujeres de una universidad privada en Arequipa. Para ello se tomó una muestra de 246 alumnos del primer semestre (117 mujeres y 129 hombres). Se aplicaron dos instrumentos: el test EQI Bar-On (Emotional Quotient Inventory), adaptado por Ugarriza y Pajares, y el Utrecht Work Engagement Scale (UWES-9), adaptado por Schaufeli y Bakker. El diseño es de tipo no experimental de nivel relacional. Los resultados indican que el promedio de inteligencia emocional obtenido en los estudiantes fue de 77,04, de 120 puntos posibles. En cuanto a la variable de engagement se alcanzó un promedio de 51.09, de 68 puntos como máximo. En cuanto a las correlaciones de la variable engagement con los componentes de la variable inteligencia emocional y con la misma variable inteligencia emocional estratificada por sexos, se observa que en las mujeres existe relación entre el engagment y los componentes interpersonales, intrapersonales, de adaptabilidad, de impresión y con la variable inteligencia emocional, en tanto que en los varones las correlaciones van desde .383 hasta .202 .

Palabras clave: Engagement; Inteligencia; Afectividad; Estudiante universitario.

\section{Summary}

The objective of this study was to determine the relationship between engagement and emotional intelligence in male and female students of a private university in Arequipa. For this, a sample of 246 students of the first semester (117 women and 129 men) was taken. Two instruments were applied: the EQI Bar-On (Emotional Quotient Inventory) test adapted by Ugarriza and Pajares and the Utrecht Work Engagement Scale (UWES-9) adapted by Schaufeli and Bakker. The design is of a non-experimental type of relational level. The results indicate that the average emotional intelligence obtained in the students was 77.04 , out of 120 possible points. Regarding the engagement variable, an average of 51.09 was reached, with a maximum of 68 points. Regarding the correlations of the engagement variable with the components of the emotional intelligence variable and with the same emotional intelligence variable stratified by sexes, it is observed that in women there is a relationship between engagment and interpersonal, intrapersonal, adaptability, impression components. and with the variable emotional intelligence, while in men the correlations range from .383 to .202 .

Keywords: Engagement; Intelligence; Emotions; University Student.

\section{Introducción}

El engagement académico se define como un estado de bienestar psicológico compuesto por tres dimensiones de compromiso intrínseco hacia el estudio: vigor (voluntad de dedicar el esfuerzo a una acción concreta y persistir ante las dificultades), dedicación (involucramiento en la tarea y la experimentación de orgullo y entusiasmo en la misma) y absorción (la concentración e inmersión en la propia acción) (Salanova, Bresó \& Schaufeli, 2005; Parra \& Pérez, 2010), variable que ha sido estudiada desde el enfoque de la psicología educativa positiva, modelo que se centra en el estudio y la promoción de los factores que promueven el comportamiento académico positivo y el bienestar psicológico (Baena-Extremera \& Granero-Gallegos, 2013; Seligman, Ernst, Guillham, Reivich, \& Linkins, 2009). Estudios anteriores han evidenciado un efecto positivo del engagement en fenómenos académicos como el rendimiento, la acumulación de capital social y el propio desenvolvimiento del estudiante en la vida universitaria (Urquijo \& Estremera, 2017). Asimismo, varios estudios han demostrado que aquellos estudiantes que tienen un mayor nivel de engagement académico presentan mayor compromiso hacia sus procesos de aprendizaje y mayor persistencia y motivación por avanzar y culminar su carrera, motivación que se da incluso durante el ejercicio de ella (Mih \& Mih, 2013; Martin, 2008; Bridges, Cambridge, Kuh \& Leegwater, 
2005; Pascarella \& Terenzini, 2005; Tinto, 2005). En ese sentido, el engagement se asocia con un comportamiento académico óptimo, en el que desempeño e integración exitosa a la vida universitaria son evidencias de sus efectos. En resumen, esta variable es una medida del involucramiento que tiene el alumno con sus estudios universitarios (McInnis et al., 2000).

El mercado actual se ha vuelto cada vez más competitivo, al punto que muchos estudiantes ya evidencian la sensación de una falta de coherencia entre lo que aprenden en la universidad y lo que exige el mercado laboral. A este fenómeno debe asociarse también la falta de empleo y la alta competitividad a la que serán sometidos los profesionales una vez insertados en el mundo laboral (Manzano, 2004). Dada esta situación, surgen diversos fenómenos que afectan el estado de bienestar psicológico del estudiante, entre los que destacan el estrés y el burnout (Extremera \& Durán, 2007; Martos et al., 2018), el rendimiento académico, la depresión, la ansiedad (Caballero-Domínguez, González \& Palacio, 2015), la deserción académica (Archambault, Janosz, Fallu y Pagani, 2009) y, claro, la inteligencia emocional. De lo expuesto, se concluye que el engagement no es un fenómeno aislado ni homogéneo entre hombres o mujeres, sino que debe ser estudiado desde su relación con otros fenómenos que permitan tener un panorama completo de los problemas que el estudiante sufre durante su vida académica.

La inteligencia emocional tiene sus bases en la obra de Goleman (1995) La inteligencia emocional, en la que la define como la forma de interacción de las personas con el mundo que las rodea. Su concepción guarda relación con la propuesta de las inteligencias múltiples de Gardner (2005), ya que entre los componentes que la integran figuran el intrapersonal (relacionado con las capacidades de identificación, percepción y dominio de las emociones en uno mismo), e interpersonal (vinculado con las capacidades de identificación y percepción de emociones en otras personas y la capacidad de relacionarnos socialmente de manera positiva) (Del Rosal, Dávila, Sánchez \& Bermejo, 2016). El componente intrapersonal se manifiesta mediante la autoconciencia y autocontrol del sujeto ante las situaciones que vive, en tanto que el interpersonal se manifiesta en la empatía y en las habilidades sociales que debe poseer el sujeto para una convivencia armónica. Una persona que posea esta inteligencia debe ser capaz de conocer las emociones del propio individuo en el momento en el que surgen; tener la capacidad de controlar las propias emociones y acomodarlas a la situación en la que se producen; reconocer no sólo las propias emociones, sino también las emociones de las personas que nos rodean, favoreciendo la empatía: mantener un control emocional, teniendo la capacidad de desarrollar la motivación; controlar, favorecer y desarrollar las relaciones personales positivas, entre otras (Del Rosal, Dávila, Sánchez \& Bermejo, 2015, p. 52)

Ser personas emocionalmente inteligentes implica aplicar las competencias emocionales en los diversos contextos en los que nos desenvolvemos. Si bien el contexto familiar fomenta el desarrollo de relaciones personales que ayudan a fomentar distintas competencias emocionales, su aplicación tiene un impacto igual de importante en la educación, en la escuela y en la universidad (Estrada et al., 2016). Es así que el estudio de las emociones no solo resulta de interés en la población de los estudiantes, sino también en los propios docentes, pues para que el alumno aprenda y desarrolle las habilidades emocionales y afectivas relacionadas con el uso inteligente de sus emociones necesita de un "educador emocional" (Extremera \& Fernández-Berrocal, 2004).

Ahora bien, en lo que respecta a su medición, existen diversos constructos que generan controversias entre los especialistas (Mayer, Roberts \& Barsade, 2008). No obstante, el modelo con mayor aceptación es el propuesto por Mayer y Salovey (1997), quienes definen la inteligencia emocional como una habilidad para percibir, asimilar, comprender y regular las emociones propias y las de los demás. Los estudios sobre inteligencia emocional en el ámbito de la educación superior han buscado determinar su relación con otras variables como son las relaciones sociales (Lopes, Salovey \& Straus, 2003; Martin-Raugh, Kell \& Motowidlo, 2016), la fatiga en el estudio (Brown \& Shutte, 2006), el bienestar subjetivo (Kong, Zhao \& You, 2012) y el éxito académico (Saklofske, Austin, Mastoras, Beaton \& Osborne, 2012) (citado en Santamaría \& Valdés, 2017). 
En lo que respecta a su relación con el engagement, los estudios han mostrado que entre estas dos variables existen relaciones significativas al compararlas según el sexo y la edad de los estudiantes (Durán, Extremera, Rey, Fernández-Berrocal \& Montalbán, 2006; Extremera, Durán \& Rey, 2007), lo cual quiere decir que si un estudiante es emocionalmente inteligente, tendrá mayor claridad sobre su carrera y manifiestará comportamientos académicos que facilitan tanto la persistencia como el engagement (Drummond, 2014) (citado en Urquijo \& Extremera, 2017).

En consecuencia, en el presente estudio se pretende valorar el engagement y la inteligencia emocional de una muestra de estudiantes hombres y estudiantes mujeres de una universidad privada en la ciudad de Arequipa, con el fin de analizar su grado de relación y sus manifestaciones, por lo que se formula la siguiente interrogante: “¿Cuál es la relación entre el engagement y la inteligencia emocional en estudiantes varones y estudiantes mujeres universitarios?"

\section{Método}

\section{Diseño}

La presente investigación es de diseño no experimental, ya que no se manipularon las variables para su análisis, y de nivel correlacional con un solo corte muestral, puesto que se analizó la asociación entre variables.

\section{Muestra}

La muestra estuvo compuesta por 246 estudiantes del primer semestre de una universidad privada en la ciudad de Arequipa. De ellos, 117 son mujeres y 129 varones. Los estudiantes pertenecen a las carreras profesionales de Arquitectura, Ingeniería Mecánica, Psicología, Ingeniería Industrial, Administración de Negocios Internacionales, Ingeniería Ambiental, Ingeniería Civil, Ingeniería de Minas y Derecho.

\section{Instrumentos}

Para la medición de la variable engagement se utilizó el instrumento Utrecht Work Engagement Scale (UWES-9) propuesta por Schaufeli y Bakker (2003). La UWES-s 9 está constituida por nueve ítems que reflejan las tres dimensiones de engagement, cada una representada por tres ítems que se evalúan a través de una escala tipo Likert, que va desde nunca (0) hasta siempre (6). La fiabilidad de la UWES-s 9, medida a través del alfa para las dimensiones de vigor, dedicación y absorción, son de $0.73,0.76$ y 0.70 , respectivamente, y 0.84 para la escala total (Schaufeli \& Bakker, 2003).

Para medir la variable de inteligencia emocional (IE) se utilizó el test EQI Bar-On (Emotional Quotient Inventory), adaptado para el Perú por Ugarriza y Pajares (2005). Este inventario permite medir un conjunto de habilidades no cognitivas, competencias y destrezas que influyen en nuestra habilidad para tener éxito en ajustarnos a las demandas y presiones del ambiente (BarOn, 1997). Es así que su uso ofrece y representa, de manera confiable, los indicadores que permiten evaluar las habilidades de las competencias emocionales como lo son las intrapersonales, interpersonales, de adaptabilidad y el estado de ánimo general.

\section{Procedimiento}

El estudio se pudo realizar gracias a los permisos otorgados por las autoridades universitarias. La ejecución de la investigación se gestionó directamente con los coordinadores de cada una de las carreras profesionales. Así mismo, una vez realizado el contacto se brindó una breve explicación del proyecto y de su alcance con el fin de fortalecer el apoyo con los estudiantes. Una vez 
obtenidos los permisos se procedió a evaluar a los estudiantes del primer semestre con ayuda de los profesores de cursos generales, quienes brindaron parte de sus horas de clases para la aplicación de los instrumentos. La aplicación de la prueba se hizo en aproximadamente 20 minutos por persona, previa explicación de los fines del estudio y de su aceptación voluntaria como participante. Las evaluaciones se realizaron de manera personal. Luego de obtenida la información, esta se exportó al programa SPSS versión 22.0 para su procesamiento, utilizando además el software GPower 3.1. La aplicación de los cuestionarios se realizó en una sola sesión y en grupo, durante las clases habituales. Se informó a los alumnos del carácter anónimo y confidencial de la información obtenida a través del cuestionario.

\section{Resultados}

En este estudio se evaluaron 246 estudiantes, de los cuales 117 (47.5\%) son mujeres, y 129 $(52.5 \%)$ son varones. El promedio de inteligencia emocional obtenido en los estudiantes es de 77.04, de 120 puntos posibles, destacando las mujeres con 77.37. Los componentes de esta variable son 1.1 Intrapersonal, 1.2 Interpersonal, 1.3 de Adaptabilidad, 1.4. de manejo del Estrés y 1.5 de Impresión o del Estado de Ánimo en General. El componente de esta variable con mayor promedio corresponde a Interpersonal en las mujeres (22.25 de 24), mientras que el indicador con menor puntaje es Manejo del estrés en las mujeres (11.67 de 20). En cuanto a la variable de engagement, se alcanzó un promedio de 51.09, de 68 puntos como máximo, destacando los varones con 52.48. Los indicadores de esta variable son: 2.1 Vigor, 2.2 Dedicación y 2.3 Absorción. El indicador con mayor puntaje de esta variable fue Dedicación, en los varones (17.19 de 20), en tanto que el indicador con menor puntuación fue Vigor, en las mujeres (16.32 de 24) (ver tabla 1).

Tabla 1.

Estadísticos descriptivos por sexos

\begin{tabular}{llccccc}
\hline Sexo & & $\mathrm{N}$ & Mínimo & Máximo & Media & $\begin{array}{c}\text { Desviación } \\
\text { estándar }\end{array}$ \\
\hline Mujer & Vigor & 117 & 6.00 & 22.00 & 16.3248 & 2.94732 \\
& Dedicación & 117 & 5.00 & 20.00 & 16.2906 & 2.98866 \\
& Absorción & 117 & 6.00 & 24.00 & 16.9316 & 2.84280 \\
& Engagment & 117 & 17.00 & 64.00 & 49.5470 & 7.43187 \\
\hline Interpersonal & 117 & 12.00 & 33.00 & 22.2479 & 4.45457 \\
& Intrapersonal & 117 & 8.00 & 23.00 & 17.4274 & 2.45763 \\
& Adaptabilidad & 117 & 10.00 & 27.00 & 18.7778 & 3.15712 \\
& Estrés & 117 & 5.00 & 19.00 & 11.6752 & 3.00525 \\
& Impresión & 117 & 3.00 & 11.00 & 7.2393 & 1.68998 \\
& Inteligencia & 117 & 48.00 & 97.00 & 77.3675 & 9.97981 \\
\hline Hombre & Vigor & 129 & 9.00 & 24.00 & 17.4496 & 2.78377 \\
& Dedicación & 129 & 11.00 & 20.00 & 17.1938 & 2.46246 \\
& Absorción & 129 & 9.00 & 24.00 & 17.8372 & 2.92562 \\
& Engagment & 129 & 34.00 & 68.00 & 52.4806 & 6.91251 \\
\hline Interpersonal & 129 & 9.00 & 32.00 & 21.9147 & 4.07552 \\
& Intrapersonal & 129 & 6.00 & 24.00 & 17.2016 & 2.79335 \\
& Adaptabilidad & 129 & 7.00 & 27.00 & 18.5426 & 3.25722 \\
& Estrés & 129 & 5.00 & 19.00 & 11.6899 & 3.13697 \\
& Impresión & 129 & 3.00 & 11.00 & 7.4031 & 1.57381 \\
& Inteligencia & 129 & 30.00 & 101.00 & 76.7519 & 10.42760 \\
\hline
\end{tabular}


Por otra parte, se realizó un análisis de normalidad de los componentes y las variables (ver Tabla 2), obteniéndose que los datos no cumplen con la prueba de normalidad, al ser menores que 0.05 ; por tanto, debe utilizarse una prueba no paramétrica para comprobar la correlación entre las variables y sus dimensiones. La prueba seleccionada es la correlación de Spearman, ya que se cumplen las dos condiciones básicas para utilizarla, que son que su nivel de medición sea de razón o intervalar y que sean no normales.

Tabla 2.

Pruebas de normalidad

\begin{tabular}{lccc}
\hline & & Kolmogorov-Smirnov $^{\mathrm{a}}$ & \\
& Estadístico & $\mathrm{gI}$ & Sig. \\
\hline Interpersonal & .066 & 246 & .011 \\
Intrapersonal & .112 & 246 & .000 \\
Adaptabilidad & .098 & 246 & .000 \\
Estrés & .098 & 246 & .000 \\
Impresión & .132 & 246 & .000 \\
Inteligencia & .053 & 246 & .047 \\
\hline Vigor & .105 & 246 & .000 \\
Dedicación & .137 & 246 & .000 \\
Absorción & .099 & 246 & .000 \\
Engagment & .075 & 246 & .002 \\
\hline
\end{tabular}

a. Corrección de significación de Lilliefors

Para determinar si los componentes de la variable Engagment guardan relación con los componentes de la variable Inteligencia emocional y si existe relación entre las variables y los componentes en hombres y mujeres, se llevó a cabo una correlación de Spearman y se obtuvo los coeficientes de correlación, la significancia estadística (sig), tamaño del efecto (p) y la potencia estadística $(1-\beta)$. Los resultados se presentan a continuación:

\section{Correlaciones de la dimensión Vigor con los componentes de la variable inteligencia emocional en función del sexo}

Respecto a las correlaciones de la dimensión Vigor con los componentes de la variable Inteligencia emocional por sexos (ver Tabla 3), se observa que en las mujeres existe relación entre el vigor y los componentes interpersonales, intrapersonales, de adaptabilidad, impresión y con la variable inteligencia emocional. Las correlaciones van desde .440 hasta .268. Los tamaños del efecto son grandes, ya que van desde .663 hasta .518 , lo cual valida la correlación y demuestra que esta es pertinente e importante. En cuanto a la potencia estadística, los valores superan a 0.80 , y se obtuvo valores que van desde 1.00 hasta .997 , lo cual demuestra que estos resultados pueden generalizarse.

En cuanto a los varones, existe relación entre el vigor y los componentes intrapersonales y de adaptabilidad, y con la variable inteligencia emocional. Las correlaciones van desde .331 hasta .263. Los tamaños del efecto son grandes, ya que van desde .575 hasta .513 , validándose también la correlación y demostrando que esta es pertinente e importante. En cuanto a la potencia estadística, los valores superan a 0.80 , y se obtuvo valores cercanos a 1.00 , por lo que estos resultados pueden generalizarse. 
Tabla 3.

Correlaciones de la dimensión Vigor con los componentes de la variable inteligencia emocional en función del sexo

\begin{tabular}{|c|c|c|c|c|c|c|c|}
\hline Sexo & & $\begin{array}{c}\text { Interperson } \\
\text { al }\end{array}$ & Intrapersonal & $\begin{array}{c}\text { Adaptabi } \\
\text { lidad }\end{array}$ & Estrés & Impresión & $\begin{array}{c}\text { Inteligenci } \\
\mathrm{a}\end{array}$ \\
\hline \multirow[t]{5}{*}{ Mujer } & $\begin{array}{l}\text { Rho de } \\
\text { Spearman }\end{array}$ & $.340 * *$ & $.268 * *$ & $.362 * *$ & .141 & $.290 * *$ & $.440 * *$ \\
\hline & $\begin{array}{l}\text { Sig. } \\
\text { (bilateral) }\end{array}$ & .000 & .003 & .000 & .129 & .001 & .000 \\
\hline & $\mathrm{p}$ & .583 & .518 & .602 & .375 & .539 & .663 \\
\hline & $1-\beta$ & 1.000 & .999 & 1.000 & .997 & .999 & .999 \\
\hline & $\mathrm{N}$ & 117 & 117 & 117 & 117 & 117 & 117 \\
\hline \multirow[t]{5}{*}{ Hombre } & $\begin{array}{l}\text { Rho de } \\
\text { Spearman }\end{array}$ & .171 & $.331^{* *}$ & $.299^{* *}$ & -.014 & .097 & $.263^{* *}$ \\
\hline & $\begin{array}{l}\text { Sig. } \\
\text { (bilateral) }\end{array}$ & .052 & .000 & .001 & .872 & .276 & .003 \\
\hline & $\mathrm{p}$ & .414 & .575 & .547 & .118 & .311 & .513 \\
\hline & $1-\beta$ & .999 & 1.000 & 1.000 & .948 & .994 & 1.000 \\
\hline & $\mathrm{N}$ & 129 & 129 & 129 & 129 & 129 & 129 \\
\hline
\end{tabular}

**. La correlación es significativa en el nivel .01 (2 colas).

*. La correlación es significativa en el nivel .05 (2 colas).

\section{Correlaciones de la dimensión Dedicación con los componentes de la variable Inteligencia emocional en función del sexo}

En cuanto a las correlaciones de la dimensión Dedicación con los componentes de la variable Inteligencia emocional por sexos (ver Tabla 4), se observa que en las mujeres existe relación entre la dedicación y los componentes interpersonales, intrapersonales, de adaptabilidad y de impresión con la variable inteligencia emocional. Las correlaciones van desde .322 hasta .188. Los tamaños del efecto son grandes y medios, ya que van desde .567 hasta .434 , quedando de esta manera validada la correlación siendo esta relevante e importante. En cuanto a la potencia estadística, los valores superan a 0.80 , y se obtuvo valores que van desde 1.00 hasta .998 , lo que demuestra que estos resultados pueden generalizarse a toda la población en estudio.

En los varones, existe relación entre la dedicación y los componentes intrapersonales y de estrés. Las correlaciones van desde .334 hasta .181. Los tamaños del efecto son grandes, ya que van desde .578 hasta .425 , lo que valida también la correlación y demuestra que esta es pertinente e importante. En cuanto a la potencia estadística, los valores superan a 0.80 , y se obtuvo valores que van desde 1.00 hasta .999 , por lo que estos resultados pueden generalizarse. 
Tabla 4.

Correlaciones de la dimensión Dedicación con los componentes de la variable Inteligencia emocional en función del sexo

\begin{tabular}{|c|c|c|c|c|c|c|c|}
\hline \multirow{6}{*}{$\frac{\text { Sexo }}{\text { Mujer }}$} & & Interpersonal & Intrapersonal & Adaptabilidad & Estrés & Impresión & Inteligencia \\
\hline & $\begin{array}{l}\text { Rho de } \\
\text { Spearman }\end{array}$ & $.188^{*}$ & $.260 * *$ & $.322 * *$ & .054 & $.212 *$ & $.321 * *$ \\
\hline & $\begin{array}{l}\text { Sig. } \\
\text { (bilateral) }\end{array}$ & .043 & .005 & .000 & .565 & .022 & .000 \\
\hline & $\mathrm{p}$ & .434 & .510 & .567 & .232 & .460 & .567 \\
\hline & $1-\beta$ & .998 & .999 & .998 & .976 & .999 & 1.000 \\
\hline & $\mathrm{N}$ & 117 & 117 & 117 & 117 & 117 & 117 \\
\hline \multirow[t]{5}{*}{ Hombre } & $\begin{array}{l}\text { Rho de } \\
\text { Spearman }\end{array}$ & .077 & $.334 * *$ & .096 & $-.181 *$ & .023 & .111 \\
\hline & $\begin{array}{l}\text { Sig. } \\
\text { (bilateral) }\end{array}$ & .386 & .000 & .310 & .040 & .798 & .210 \\
\hline & $\mathrm{p}$ & .277 & .578 & .293 & .425 & .152 & .333 \\
\hline & $1-\beta$ & .990 & 1.000 & .995 & .999 & .953 & .996 \\
\hline & $\mathrm{N}$ & 129 & 129 & 129 & 129 & 129 & 129 \\
\hline
\end{tabular}

** La correlación es significativa en el nivel .01 ( 2 colas).

* La correlación es significativa en el nivel .05 (2 colas).

\section{Correlaciones de la dimensión Absorción con los componentes de la variable Inteligencia emocional en función del sexo}

En cuanto a las correlaciones de la dimensión Absorción con los componentes de la variable inteligencia emocional por sexos (ver tabla 5), se observa que en las mujeres existe relación entre la absorción y los componentes interpersonales, intrapersonales, de adaptabilidad y de impresión con la variable Inteligencia emocional. Las correlaciones van desde .328 hasta .250. Los tamaños del efecto son grandes, ya que van desde .573 hasta .500 , lo que valida la correlación y demuestra que esta es pertinente e importante. En cuanto a la potencia estadística, los valores superan a 0.80 y se obtuvo valores que van desde 1.00 hasta .999 , lo cual demuestra que estos resultados pueden generalizarse.

En los varones, existe relación entre la absorción y los componentes intrapersonales, intrapersonales y de adaptabilidad con la variable Inteligencia emocional. Las correlaciones van desde .331 hasta .262 . Los tamaños del efecto son grandes, ya que van desde .575 hasta .512 , lo cual valida también la correlación y demuestra que esta es pertinente e importante. En cuanto a la potencia estadística, los valores superan a 0.80 y se obtuvo valores de 1.00 , por lo que estos resultados pueden generalizarse. 
Tabla 5.

Correlaciones de la dimensión Absorción con los componentes de la variable Inteligencia emocional en función del sexo

\begin{tabular}{|c|c|c|c|c|c|c|c|}
\hline Sexo & & $\begin{array}{c}\text { Intraperso } \\
\text { nal }\end{array}$ & $\begin{array}{c}\text { Interperson } \\
\text { al }\end{array}$ & $\begin{array}{c}\text { Adaptabilida } \\
\mathrm{d}\end{array}$ & Estrés & $\begin{array}{c}\text { Impresió } \\
n\end{array}$ & $\begin{array}{c}\text { Inteligenci } \\
\mathrm{a}\end{array}$ \\
\hline \multirow[t]{5}{*}{ Mujer } & $\begin{array}{l}\text { Rho de } \\
\text { Spearman }\end{array}$ & $.254 * *$ & $.254 * *$ & $.274 * *$ & .083 & $.250 * *$ & $.328 * *$ \\
\hline & $\begin{array}{l}\text { Sig. } \\
\text { (bilateral) }\end{array}$ & .006 & .006 & .003 & .372 & .006 & .000 \\
\hline & $\mathrm{p}$ & .504 & .504 & .523 & .288 & .500 & .573 \\
\hline & $1-\beta$ & .999 & .999 & .999 & .989 & .999 & 1.000 \\
\hline & $\mathrm{N}$ & 117 & 117 & 117 & 117 & 117 & 117 \\
\hline \multirow[t]{5}{*}{ Hombre } & $\begin{array}{l}\text { Rho de } \\
\text { Spearman }\end{array}$ & $.262 * *$ & $.296 * *$ & $.331 * *$ & .035 & .017 & $.290 * *$ \\
\hline & $\begin{array}{l}\text { Sig. } \\
\text { (bilateral) }\end{array}$ & .003 & .001 & .000 & .691 & .845 & .001 \\
\hline & $\mathrm{p}$ & .512 & .544 & .575 & .187 & .130 & .539 \\
\hline & $1-\beta$ & 1.000 & 1.000 & 1.000 & .965 & .948 & 1.000 \\
\hline & $\mathrm{N}$ & 129 & 129 & 129 & 129 & 129 & 129 \\
\hline
\end{tabular}

* La correlación es significativa en el nivel .05 (2 colas).

** La correlación es significativa en el nivel .01 (2 colas).

\section{Correlaciones de la variable Engagement con los componentes de la variable Inteligencia emocional en función del sexo}

En cuanto a las correlaciones de la variable Engagement con los componentes de la variable Inteligencia emocional y con la misma variable Inteligencia emocional estratificada por sexos (ver tabla 6), se observa que en las mujeres existe relación entre el engagement y los componentes interpersonales, intrapersonales, de adaptabilidad, de impresión con la variable Inteligencia emocional. Las correlaciones van desde .444 hasta .326. Los tamaños del efecto son grandes, ya que van desde ,666 hasta .551, lo que valida la correlación y demuestra que esta es pertinente e importante. En cuanto a la potencia estadística, los valores superan a 0.80 y se obtuvo valores de 1.00 , lo que demuestra que estos resultados pueden generalizarse.

En los

varones, existe relación entre el engagement y los componentes intrapersonales, intrapersonales y de adaptabilidad con la variable Inteligencia emocional. Las correlaciones van desde .383 hasta .202. Los tamaños del efecto son grandes, ya que van desde .619 hasta .557 , lo que valida también la correlación y demuestra que esta es relevante e importante. En cuanto a la potencia estadística, los valores superan a 0.80 y se obtuvo valores de 1.00 , por lo que estos resultados pueden generalizarse. 
Tabla 6.

Correlaciones de la variable Engagment con los componentes de la variable Inteligencia emocional en función del sexo

\begin{tabular}{|c|c|c|c|c|c|c|c|}
\hline Sexo & & $\begin{array}{c}\text { Interperso } \\
\text { nal }\end{array}$ & $\begin{array}{c}\text { Intraperson } \\
\text { al } \\
\end{array}$ & $\begin{array}{c}\text { Adaptabilida } \\
\text { d }\end{array}$ & Estrés & $\begin{array}{c}\text { Impresió } \\
\mathrm{n}\end{array}$ & $\begin{array}{c}\text { Inteligenci } \\
\mathrm{a}\end{array}$ \\
\hline \multirow[t]{5}{*}{ Mujer } & $\begin{array}{l}\text { Rho de } \\
\text { Spearman }\end{array}$ & $.331 * *$ & $.326 * *$ & $.386^{* *}$ & .106 & $.304 * *$ & $.444 * *$ \\
\hline & $\begin{array}{l}\text { Sig. } \\
\text { (bilateral) }\end{array}$ & .000 & .000 & .000 & .254 & .001 & .000 \\
\hline & $\mathrm{p}$ & .575 & .571 & .621 & .326 & .551 & .666 \\
\hline & $1-\beta$ & 1.000 & 1.000 & 1.000 & .993 & 1.000 & 1.000 \\
\hline & $\mathrm{N}$ & 117 & 117 & 117 & 117 & 117 & 117 \\
\hline \multirow[t]{5}{*}{$\begin{array}{l}\text { Hombr } \\
\mathrm{e}\end{array}$} & $\begin{array}{l}\text { Rho de } \\
\text { Spearman }\end{array}$ & $.202 *$ & $.383 * *$ & $.310 * *$ & -.040 & .062 & $.270 * *$ \\
\hline & $\begin{array}{l}\text { Sig. } \\
\text { (bilateral) }\end{array}$ & .022 & .000 & .000 & .656 & .484 & .002 \\
\hline & $\mathrm{p}$ & .449 & .619 & .557 & .200 & .249 & .520 \\
\hline & $1-\beta$ & .999 & 1.000 & 1.000 & .970 & .985 & 1.000 \\
\hline & $\mathrm{N}$ & 129 & 129 & 129 & 129 & 129 & 129 \\
\hline
\end{tabular}

** La correlación es significativa en el nivel .01 (2 colas).

* La correlación es significativa en el nivel .05 (2 colas).

\section{Discusión}

La presente investigación busca determinar la relación entre el engagement académico y la inteligencia emocional en estudiantes hombres y estudiantes mujeres en una universidad privada en Arequipa. Así, los resultados obtenidos aportan evidencia empírica sobre la manera en que se relacionan estas variables. De manera consistente con estudios previos realizados y registrados en la literatura, el presente trabajo encontró que una mayor inteligencia emocional por parte de los estudiantes universitarios se relacionaba positivamente con el engagement académico (Urquijo \& Extremera, 2017; Serrano \& Andreu, 2017; Esteban, 2014; Peña, Rey \& Extremera, 2012). Con respecto a los análisis descriptivos, el promedio de inteligencia emocional obtenido en los estudiantes fue de 77.04, de 120 puntos posibles. En cuanto a la variable de engagement se alcanzó un promedio de 51.09, de 68 puntos como máximo. Estos resultados, coinciden con los hallados por Urquijo y Extremera (2017), quienes trabajaron con una muestra de 203 estudiantes de la Universidad de Deusto con edades comprendidas entre 18 y 27 años, muy similar a la utilizada en la presente investigación.

El engagement debe entenderse como "percibir el trabajo como una experiencia estimulante y destinando tiempo y esfuerzo (el componente vigor); en una actividad significativa y valiosa (dedicación) y como algo interesante y apasionante (absorción)" (Bakker, Demerouti \& Xanthopoulou, 2011, p.136). Este concepto corrobora la implicación que tienen los procesos emocionales en aspectos como el esfuerzo (vigor), entusiasmo (dedicación) y la entrega (absorción) de los estudiantes como parte de su trabajo diario en la universidad. Esto queda corroborado en diversas investigaciones (Zuloaga \& Moreno, 2012) que indican que los estudiantes que reconocen y expresan sus emociones presentan niveles elevados de energía, dedicación y entrega en sus labores académicas.

En cuanto a los resultados por sexo, se observa que en las mujeres existe un mayor coeficiente de relación que entre los hombres. Las correlaciones del primer grupo van desde .444 hasta .326, en cuanto que, en los varones, las correlaciones van desde .383 hasta .202. Los tamaños 
del efecto son grandes y similares en ambos sexos, ya que van desde .619 hasta .557 , lo que valida la correlación y demuestra que esta es pertinente e importante. En cuanto a la potencia estadística, los valores superan a 0.80 y se obtuvo valores de 1.00 , lo que demuestra que estos resultados pueden generalizarse.

Estos resultados revelaron una relación positiva entre las dimensiones de la inteligencia emocional, excepto el de manejo del estrés en ambos sexos y de impresión o estado de ánimo en general en el caso de los hombres. Sin embargo, al analizar las relaciones entre las dimensiones del engagment y la inteligencia emocional, esta se relaciona significativamente con el vigor en ambos sexos, la dedicación sólo en mujeres y la absorción en los dos sexos. Otras investigaciones también mostraron que la inteligencia emocional, en este caso de trabajadores, desarrolla un compromiso con la institución (Humphreys et al., 2005; Andrade, Santos \& Franco, 2016).

Finalmente, al analizar las relaciones entre las dimensiones de la variable Engagment y los componentes de la variable Inteligencia emocional, se observa que la dimensión Vigor en las mujeres guarda relación con los componentes interpersonales, intrapersonales, de adaptabilidad y de impresión con la variable Inteligencia emocional. En cuanto a los varones, existe relación con los componentes intrapersonales y de adaptabilidad con la variable Inteligencia emocional. En cuanto a la dimensión Dedicación, se observa que en las mujeres existe relación con los componentes interpersonales, intrapersonales, de adaptabilidad de impresión con la variable Inteligencia emocional. En cuanto a los varones, existe relación con los componentes intrapersonales y de estrés. Y en cuanto a las correlaciones de la dimensión Absorción, se observa que en las mujeres existe relación entre la absorción y los componentes interpersonales, intrapersonales, de adaptabilidad y de impresión con la variable inteligencia emocional. En cuanto a los varones, existe relación con los componentes intrapersonales, interpersonales y de adaptabilidad con la variable Inteligencia emocional. Queda claro que las mujeres presentan una mayor relación entre dimensiones y componentes de las variables, destacando su mayor inteligencia emocional sobre todo en su componente interpersonal, pese a que los hombres tienen un mejor engagement, destacando en la dimensión Dedicación.

Estos resultados discrepan de lo afirmado por Pérez et al. (2018) con respecto a las diferencias entre hombres y mujeres, concluyendo que las mujeres se presentan como más enérgicas y con mayor dedicación al estudio y se encuentran más absortas en sus tareas, en comparación con los hombres, es decir, presentan un mejor engagement académico. Sin embargo, Carrasco (2017) corrobora que las mujeres presentan niveles más elevados de inteligencia emocional, ya que las mujeres, en comparación con los varones, presentan más habilidad cuando se trata de percibir expresiones emocionales faciales. Asimismo, las mujeres presentan mayor empatía, lo que facilita su habilidad para reconocer emociones en otros y en sí mismas y también les permite ser más expresivas, contar con mayor facilidad de adaptarse y tener mayor facilidad para emplear la información emocional que reciben.

A pesar de aportar aspectos novedosos y trascendentes, el estudio presenta dos limitaciones. En primer lugar, los estudiantes de la muestra tienen como procedencia una sola universidad, y el muestreo ha sido realizado por conveniencia. Se recomienda que futuras investigaciones debiesen trabajar con una muestra procedente de diferentes universidades públicas y privadas. En segundo aspecto, el utilizar un diseño transversal no permite realizar una investigación explicativa causal. Por tanto, se recomienda que las futuras investigaciones sean longitudinales y experimentales, a lo que ha de sumarse una muestra más grande y una triangulación entre las fuentes de información primaria, para así corroborar de mejor manera los resultados obtenidos, que podrán inferirse de mejor manera. 


\section{Referencias}

Andrade, C., Santos, N., \& Franco, M. (2016). Inteligência emocional e engagement em professores do ensino básico e secundário da Ilha da Madeira. Revista De Estudios E Investigación En Psicología y Educación, 3(2), 121-130. doi: https://doi.org/10.17979/reipe.2016.3.2.1810

Archambault, I., Janosz, M., Fallu, J.S., \& Pagani, L.S. (2009). Student engagement and its relationship with early high school dropout. Journal of Adolescence, 32, 651-670. doi: https://doi.org/10.1016/j.adolescence.2008.06.007

Baena-Extremera, A., \& Granero-Gallegos, A. (2013). Efecto de un programa de Educación de Aventura en la orientación al aprendizaje, satisfacción y autoconcepto en secundaria. Revista Iberoamericana de Diagnóstico y Evaluación Psicológica, 36(2), 163-187.

Bakker, A.B., Demerouti, E. \& Xanthopoulou, D. (2011). ¿Cómo los Empleados Mantienen su Engagement en el Trabajo? Ciencia y trabajo, 13(41), 135-142.

BarOn, R. (1997). Development of the Baron EQ-I: A measure of emotional and social intelligence. 105th Annual Convention of the American Psychological Association in Chicago.

Bridges, B. K., Cambridge, B., Kuh, G. D. y Leegwater, L. H. (2005). Student engagement at minority-serving institutions: Emerging lessons from the BEAMS project. En G. H. Gaither (Eds.). What works: Achieving success in minority retention, (pp. 25- 43). New Directions for Institutional Research. San Francisco: Jossey-Bass. doi. https://doi.org/10.1002/ir.137

Brown R.F. \& Schutte N.S. (2006) Direct and indirect relationships between emotional intelligence and subjective fatigue in university students. Journal of Psychosomatic Research, 60, 585-593. doi: https://doi.org/10.1016/j.jpsychores.2006.05.001

Caballero-Dominguéz, C., González, O. \& Palacio, J. (2015). Relación del burnout y el engagement con depresión, ansiedad y rendimiento académico en estudiantes universitarios. Revista Científica Salud Uninorte, 31(1), 59-69. doi: https://doi.org/10.14482/sun.31.1.5085

Carrasco, J. (2017). Relación entre inteligencia emocional, autoeficacia general y variables sociodemográficas en pacientes ambulatorios del Servicio de psicología de una clínica de Lima metropolitana. Recuperado de: repositorio.upch.edu.pe/bitstream/handle/.../Relacion_CarrascoTacuri_Jennifer.pdf?

Del Rosal, I., Dávila, M., Sánchez, S. \& Bermejo, M. (2016). La inteligencia emocional en estudiantes universitarios: diferencias entre el grado de maestro en educación primaria y los grados en ciencias International Journal of Developmental and Educational Psychology, 2(1), 51-61. doi: https://doi.org/10.17060/ijodaep.2016.n1.v2.176

Durán, A., Extremera, N., Rey, L., Fernández-Berrocal, P., \& Montalbán, M. (2006). Predicting academic burnout and engagement in educational settings: assessing the incremental validity of perceived emotional intelligence beyond perceived stress and general selfefficacy. Psicothema, 18(1), 158-164. doi: https://doi.org/10.1037/t01392-000

Esteban, B. (2014). Una aproximación a la influencia de la Inteligencia Emocional Percibida en su relación con los niveles de Burnout y Engagement en el desempeño del Trabajo Social. AZARBE, Revista Internacional de Trabajo Social y Bienestar, 3, 123-131.

Estrada, E. et al. (2016). Inteligencia emocional en estudiantes universitarios mexicanos. Recuperado de http://ri.uaemex.mx/bitstream/handle/20.500.11799/65573/Interpsiquis\%202016\%20Int eligencia\%20emocional $\% 20 \mathrm{en} \% 20 \mathrm{j} \% \mathrm{c} 3 \% \mathrm{~b} 3$ venes.pdf?sequence $=1 \&$ isAllowed=y

Extremera, N. \& Durán, A. (2007). Inteligencia emocional y su relación con los niveles de burnout, engagement y estrés en estudiantes universitarios. Revista de Educación, 342, 239-256.

Extremera, N., Durán, A. \& Rey, L. (2007). Perceived emotional intelligence and dispositional optimism-pessimism: Analyzing their role in predicting psychological adjustment among 
adolescents. Personality and Individual Differences, 42(6), 1069-1079. doi: 10.1016/j.paid.2006.09.014. doi: https://doi.org/10.1016/j.paid.2006.09.014

Extremera, N., \& Fernández-Berrocal, P. (2004). La inteligencia emocional: Métodos de evaluación en el aula. Revista Iberoamericana de Educación, 34(1), 1-12. doi: https://doi.org/10.35362/rie3412887

Gardner, H. (2005). Universidad de Harvard Inteligencias múltiples. Revista de Psicología y Educación, 1(1), 17-16

Goleman, D. (1996). Inteligencia emocional. Barcelona: Kairós.

Humphreys, J., Brunsen, B., \& Davis, D. (2005). Estructura emocional y compromiso: implicaciones para la gestión del cuidado de la salud. Journal of Health Organization and Management, 19(2), 120-129. doi: https://doi.org/10.1108/14777260510600040

Kong, F., Zhao, J., \& You, X. (2012). Emotional intelligence and life satisfaction in Chinese university students: The mediating role of self-esteem and social support. Personality and Individual Differences, 53(8), 1039-1043. doi: https://doi.org/10.1016/j.paid.2012.07.032

Lopes, P. N., Salovey, P., \& Straus, R. (2003). Emotional intelligence, personality and the perceived quality of social relationships. Personality and Individual Differences, 35(1), 641-658. doi: https://doi.org/10.1016/S0191-8869(02)00242-8

Manzano, G. (2004). Perfil de los estudiantes comprometidos con sus estudios: influencia del burnout y el engagement. Anuario de Psicología, 35(4), 299-415.

Martin, A. J. (2008). Enhancing student motivation and engagement: The effects of a multidimensional intervention. Contemporary Educational Psychology, 33, 239-269. doi: https://doi.org/10.1016/j.cedpsych.2006.11.003

Martin-Raugh, M. P., Kell, H. J. \& Motowidlo, S. J. (2016). Prosocial knowledge mediates effects of agreeableness and emotional intelligence on prosocial behavior. Personality and Individual Differences, 90, 41-49. doi: https://doi.org/10.1016/j.paid.2015.10.024

Martos, A., Pérez-Fuentes, M., Molero., M., Gázquez, J., Simón, M., \& Barragán, A. (2018). Burnout y engagement en estudiantes de Ciencias de la Salud. European Journal of Investigation in Health, Psychology and Education, 8(1), 23-36). doi: https://doi.org/10.30552/ejihpe.v8i1.223

Mayer, J.D., Roberts, R.D. \& Barsade, S.G. (2008). Human abilities: emotional intelligence. The Annual Review of Psychology, 59(1), 507-536. doi: https://doi.org/10.1146/annurev.psych.59.103006.093646

Mayer, J. D. \& Salovey, P. (1997). What is emotional intelligence? En P. Salovey y D. Sluyter (Eds.), Emotional development and emotional intelligence: implications for educators (pp. 3-34). New York: Basic Books.

McInnis, C., James, R., \& Hartley, R. (2000). Trends in the firstyear experience. Canberra: DETYA. Higher Education Division.

Mih, V., \& Mih, C. (2013). Perceived autonomy-supportive teaching, academic self-perceptions and engagement in learning: Toward a process model of academic achievement. Cognition, Brain \& Behavior. An Interdisciplinary Journal, 4, 289-313.

Parra, P. \& Pérez, C. (2010). Propiedades psicométricas de la escala de compromiso académico, UWES-S (versión abreviada), en estudiantes de psicología. Revista de Educación de Ciencias de la Salud, 7(1), 128-133.

Pascarella, E. T. \& Terenzini, P. T. (2005). How college affects students: A Third Decade of Research. San Francisco: John Wiley and Sons.

Peña, M., Rey, L., \& Extremera, N. (2012). Life Satisfaction and Engagement in Elementary and Primary Educators: Differences in Emotional Intelligence and Gender. Revista de Psicodidáctica, 17(2), 341-360. doi: https://doi.org/10.1387/RevPsicodidact.1220

Pérez, M. C., Molero, M. M., Barragán, A. B., Martos, A., Simón, M. M., \& Gázquez, J. J. (2018). Autoeficacia y engagement en estudiantes de Ciencias de la Salud y su relación con la autoestima. $\quad$ Publicaciones, $48(1), \quad 161-172 . \quad$ doi: https://doi.org/10.30827/publicaciones.v48i1.7323

Santamaría, B. \& Valdés, M $^{\mathrm{a}}$. (2017). Rendimiento del alumnado de educación secundaria obligatoria: influencia de las habilidades sociales y la inteligencia emocional. 
International Journal of Developmental and Educational Psychology, 2(1), 57-66. Doi: https://doi.org/10.17060/ijodaep.2017.n1.v2.918

Salanova, M., Bresó, E., \& Schaufeli, W. B. (2005). Hacia un modelo espiral de las creencias de eficacia en el estudio del burnout y del engagement. Ansiedad y estrés, 11(2-3), 215-231.

Schaufeli, W., \& Bakker, A. (2003). uwes Utrecht Work Engagement Scale. Utrecht: Utrecht University. doi: https://doi.org/10.1037/t07164-000

Serrano, C., \& Andreu, Y. (2016). Inteligencia emocional percibida, bienestar subjetivo, estrés percibido, compromiso y logro académico de los adolescentes. Revista de Psicodidáctica, 21(2), 357-374. doi: https://doi.org/10.1387/RevPsicodidact.14887

Seligman, M., Ernst, R., Guillham, J., Reivich, K., \& Linkins, M. (2009). Positive education: Positive Psychology and classroom interventions. Oxford Review of Education, 35, 293311. doi: https://doi.org/10.1080/03054980902934563

Serrano, C., \& Andreu, Y. (2016). Inteligencia emocional percibida, bienestar subjetivo, estrés percibido, compromiso y logro académico de los adolescentes. Revista de Psicodidáctica, 21(2), 357-374. doi: https://doi.org/10.1387/RevPsicodidact.14887

Tinto, V. (2005). Reflections on retention and persistence: Institutional actions on behalf of student persistence. Studies in Learning, Evaluation, Innovation and Development. 2(3), 89-97.

Ugarriza, N., \& Pajares, L. (2005). La evaluación de la inteligencia emocional a través del inventario de BarOn ICE: NA, en una muestra de niños y adolescentes. Persona, 8, 1158. doi: https://doi.org/10.26439/persona2005.n008.893

Urquijo, I., \& Extremera, N. (2017). Satisfacción académica en la universidad: relaciones entre inteligencia emocional y engagement académico. Electronic Journal of Research in Educational Psychology, 15(3), 553-573. doi: https://doi.org/10.14204/ejrep.43.16064

Zuluaga, P., \& Moreno, S. (2012). Relación entre síndrome de burnout, estrategias de afrontamiento y engagement. Psicología desde El Caribe, 29(1), 205-227. 